\title{
A B-Spline Element Method for Predicting the Hydroelastic Responses of a Very Large Floating Structure in Waves
}

\author{
by Xin Lin*, Member Mikio Takaki*, Member
}

\begin{abstract}
Summary
A new modal analysis method, the B-spline element method, has been developed in this study for estimating the hydroelastic responses of a very large floating structure in waves. A motion equation for the elastic structure has been proposed in this method according to the energy-conservation principle. The unknown deflection and load, the hydrodynamic pressure, of the structure are both discretized with bi-cubic B-spline function. The B-spline expressions of the dry-eigenmodes of the structure are obtained by solving the eigenvalue problem of the proposed motion equation. With the resultant eigenmodes, hydrodynamic pressures due to the structure motion and incident waves are calculated by applying the pressure distribution method. Differing from the conventional Finite Element Method, B-spline element method ensures the continuity for not only the structure deflection but also the strains and stresses, and therefore it may have better accuracy. The usage of B-spline discretization for the pressures makes it possible to predict the hydroelastic responses in very short wavelength case. Comparisons between the calculations of the proposed method and experimental data have been carried out, and it shows that they are in a very good agreement.
\end{abstract}

\section{Introduction}

In the past two years, many studies have been reported on wave induced motions of a very large floating structure (VLFS). We may classify them into two kinds, the direct method and modal analysis method. Mamidipudi \& Webster's ${ }^{7)}$ and Ohkusu \& Nanba's ${ }^{3)}$ studies are the representative ones of the direct method. The majority of the studies are of the second kind. The first modal analysis study for a very large floating structure has been done by Maeda $e t a l^{6)}$. Takaki \& Gu's ${ }^{4)}$ and Kashiwagi \& Furukawa's ${ }^{1)}$ studies may consider be the representative ones of the three dimensional modal analysis method. In Takaki's study, dry-eigenmodes of a free-free elastic plate are used with the pressure distribution method. The problem is solved in three steps. They first determine the eigenmodes by applying finite element method (FEM). Second, they estimate the hydrodynamic pressure according to the resultant eigenmodes. Finally, they transform the pressures into nodal forces and bring the nodal forces back to the finite element calculation to obtain the amplitudes of each eigenmodes. A program of finite element method for structural analysis is necessary in this procedure. Prediction of wave-induced motion by this method has a quite good accuracy. However the method has a weak point. This method is based the constant panel discretization for the unknown pressure distribution, therefore it is difficult to apply it to the short wave case. In Kashiwagi's method, "generalized mode functions" are used along longitudinal and lateral directions to represent the deformation of the plate. The mode functions are in fact the eigenmodes of a free beam and have analytical expressions. Hydrodynamic pressures are also solved by applying the pressure distribution method but with bi-cubic B-spline panels. Amplitudes

\footnotetext{
* Faculty of Engineering, Hiroshima University
}

Received 9th Jan. 1998

Read at the Spring meeting 14, 15th May 1998 of the generalized modes are determined from the vibration equation of the plate, a fourth order differential equation, by applying Galerkin scheme with the generalized modes as the weight function. The advantages of this method are that it is independent from any structural analysis procedure because the generalized mode functions have analytical expressions and the usage of high order B-spline function makes it possible to calculate the very short incident wave case. Comparison of the deflections between the experimental data and the calculations by this method showed a very good agreement. While some problems might occur in calculations of the internal forces of the structure because generalized modes used in this method do not satisfy the edge condition of a free-free plate.

In this study, we have developed a new modal analysis method, B-spline element method (BSEM), for predicting the wave-induced motion of a very large floating structure. In our method, a motion equation for a elastic structure has been proposed according to the energy-conservation principle. The unknown deflection and load, the hydrodynamic pressure, of the structure are both discretized with bi-cubic B-spline function. The B-spline expressions of the dry-eigenmodes of the structure are obtained by solving the eigenvalue problem of the proposed motion equation. With the resultant eigenmodes, hydrodynamic pressures due to the structure motion and incident waves are calculated by applying the pressure distribution method. Differing from conventional finite element method, B-spline element method ensures the continuity for not only the structure deflection but also the strains and stresses, and therefore it may have better accuracy. The usage of B-spline discretization for hydrodynamic pressures makes it possible to predict the hydroelastic responses in very short wavelength case. Comparisons between the calculations of the proposed method and experimental data have been carried out for the deformations and bending moment, and it shows that the theoretical predictions and experimental results are in a very good agreement. 


\section{Motion Equation}

To obtain the motion equation, we apply Lagrange's equations to the structure. Lagrange's equations represent the energy conservation principle of the structure and have general forms of

$$
\frac{d}{d t}\left(\frac{\partial \mathcal{T}}{\partial \dot{\mathbf{q}}^{r}}\right)-\frac{\partial \mathcal{T}}{\partial \mathbf{q}^{r}}+\frac{\partial \mathcal{U}}{\partial \mathbf{q}^{r}}=\mathcal{F}_{r}
$$

where

$$
\begin{aligned}
& \mathrm{q}^{r}: \text { the } r \text {-th generalized coordinate, } \\
& \mathcal{T}: \text { kinetic energy of the structure, } \\
& \mathcal{U}: \text { deformation potential energy of the structure, } \\
& \mathcal{F}_{\boldsymbol{r}}: \text { the } r \text {-th generalized force. }
\end{aligned}
$$

If we denote the deflection vector of the structure as

$$
\boldsymbol{W}(\boldsymbol{r}, t)=\sum_{\boldsymbol{r}} \mathrm{q}^{r}(t) \boldsymbol{W}_{\boldsymbol{r}}(\boldsymbol{r}),
$$

we have the kinetic energy and generalized hydrodynamic force

$$
\begin{aligned}
\mathcal{T} & =\frac{1}{2} \iiint_{V} \varrho\left(\frac{d \boldsymbol{W}}{d t} \cdot \frac{d \boldsymbol{W}}{d t}\right) d v \\
& =\frac{1}{2} \sum_{r} \sum_{s} M_{r s} \dot{\boldsymbol{q}}^{r} \dot{\mathrm{q}}^{s} \\
\mathcal{F}_{\boldsymbol{r}} & =-\iint_{\boldsymbol{s}_{h}} P(\boldsymbol{r}, t) \boldsymbol{n} \cdot \boldsymbol{W}_{r} d s
\end{aligned}
$$

where $\varrho$ is the density of the structure, $P$ the hydrodynamic pressure on wetted surface $S_{h}$ of the structure, and $n$ the normal vector points inward the fluid domain. With stress tensor $\sigma^{i j}$ and strain tensor $\epsilon_{i j}$, we have the deformation potential energy

$$
\mathcal{U}=\frac{1}{2} \iiint_{V} \sigma^{i j} \epsilon_{i j} d v=\frac{1}{2} \sum_{r} \sum_{s} K_{r s} \mathrm{q}^{r} \mathrm{q}^{s} .
$$

The summation convention of tensor is used in the integrand. $\epsilon_{i j}$ and $\sigma^{i j}$ are functions of the deflection $\boldsymbol{W}(\boldsymbol{r}, t)$, and they satisfy the generalized Hook's law

$$
\sigma^{i j}=E^{i j k l} \epsilon_{k l},
$$

where $E^{i j k l}$ is the tensor of Young's modulus.

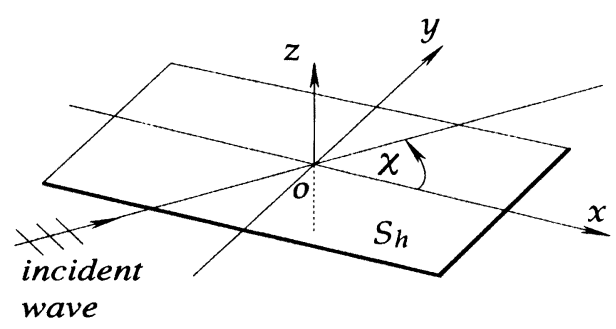

Fig. 1. Coordinate system and notations
The structure considered in this study is a floating structure with very shallow draft. Therefore we can simplify the structure to an elastic plate. The displacement of the plate along horizontal direction will be small in comparison with that along the vertical direction and be ignored, therefore $\boldsymbol{W}(x, y ; t)=W(x, y ; t) \boldsymbol{e}_{z}$. Time-harmonic elastic motions of the plate will be induced by regular incident waves. Assuming the existence of potential flow for the fluid field, we can express the velocity potential $\Phi$, pressure distribution $I^{\prime}$ and vertical deflection $W$ in following forms

$$
\begin{aligned}
\Phi(\boldsymbol{r}, t) & =\Re\left[i \omega \zeta_{a} \phi(\boldsymbol{r}) e^{i \omega t}\right], \\
P(x, y ; l) & =\Re\left[\rho g \zeta_{a} p(x, y) e^{i \omega t}\right], \\
W(x, y ; l) & =\Re\left[\zeta_{a} w(x, y) e^{i \omega t}\right],
\end{aligned}
$$

where $\boldsymbol{r}=(x, y, z), \omega$ and $\zeta_{a}$ are the circular frequency and the amplitude of the incident wave respectively, $\rho$ the fluid density and $g$ the gravitational acceleration. Their spatial components are given by

$$
\begin{aligned}
\phi & =\phi_{I}(\boldsymbol{r})+\phi_{D}(\boldsymbol{r})+\sum_{r} \frac{q^{r}}{\zeta_{a}} \phi_{r}(\boldsymbol{r}), \\
p & =p_{I}(x, y)+p_{D}(x, y)+\sum_{r} \frac{q^{r}}{\zeta_{a}} p_{r}(x, y), \\
w & =w_{I}(x, y)+w_{D}(x, y)+\sum_{r} \frac{q^{r}}{\zeta_{a}} w_{r}(x, y) .
\end{aligned}
$$

Suffix $I$ represents the quantities with respective to the incident wave, and $D$ to the scattering component. $w_{r}$ is the shape function of the $r$-th eigenmode, $q^{r}$ its complex amplitude, and $p_{r}$ the radiation pressure on the bottom due to the motion of the $r$-th eigenmode with unit amplitude. Using these definitions together with isotropic plate assumption, we have generalized mass $M_{r s}$ and stiffness $K_{r s}$

$$
\begin{aligned}
M_{r s}=\varrho d \iint_{S_{h}} w_{r}(x, y) w_{s}(x, y) d s, \\
K_{r s}=D \iint_{S_{h}}\left\{\frac{\partial^{2} w_{r}}{\partial x^{2}} \frac{\partial^{2} w_{s}}{\partial x^{2}}+\frac{\partial^{2} w_{r}}{\partial y^{2}} \frac{\partial^{2} w_{s}}{\partial y^{2}}\right. \\
+\nu\left(\frac{\partial^{2} w_{r}}{\partial x^{2}} \frac{\partial^{2} w_{s}}{\partial y^{2}}+\frac{\partial^{2} w_{r}}{\partial y^{2}} \frac{\partial^{2} w_{s}}{\partial x^{2}}\right) \\
\left.+2(1-\nu) \frac{\partial^{2} w_{r}}{\partial x \partial y} \frac{\partial^{2} w_{s}}{\partial x \partial y}\right\} d s,
\end{aligned}
$$

where $D=E d^{3} / 12\left(1-\nu^{2}\right)$ is the flexural rigidity of the plate, $E$ the Young's modulus, $\nu$ the Poisson's ratio, and $d$ the thickness of the plate. The final form of motion equation of the elastic plate for determining the amplitude of $r$-th mode is

$$
\sum_{s}\left(-K \frac{M_{r s}}{\rho}+\frac{K_{r s}}{\rho g}-\iint_{S_{h}} w_{r} p_{s} d s\right) \frac{q^{s}}{\zeta_{a}}=\iint_{S_{h}} w_{r} p_{D} d s,
$$

where $K=\omega^{2} / g$ is wave number of the incident wave. Effect of structural damping has been ignored in this equation. After obtaining the amplitudes, we can estimate the structure loads. For example, bending moments $M_{x}$ and $M_{y}$ can be evaluated with the resultant vertical deflection like

$$
\left.\begin{array}{l}
M_{x}=-D\left(\frac{\partial^{2} W}{\partial x^{2}}+\nu \frac{\partial^{2} W}{\partial y^{2}}\right) \\
M_{y}=-D\left(\frac{\partial^{2} W}{\partial y^{2}}+\nu \frac{\partial^{2} W}{\partial x^{2}}\right)
\end{array}\right\} .
$$

To solve motion equation (15), of course, we have to estimate 
the eigenmodes and the pressure distributions first.

\section{Dry-Eigenmodes}

In this method, the plate is discretized into a number of B-spline elements. The unknown vertical deflection $W$ is represented by fourth order (bi-cubic) B-spline functions. The deflection vector is given in a form of

$$
\boldsymbol{W}(x, y ; l)=\Re\left[\zeta_{a} \sum_{l} \alpha^{l} B_{l}(x, y) e^{i \omega t}\right] e_{z},
$$

where $B_{l}(x, y)$ is the product of B-spline basis functions with respective to $x$ and $y$, and $\alpha^{l}$ the unknown polygon vector. Substituting this expression into Lagrange's equation (1) and omitting the forcing term $\mathcal{F}_{l}$, we get the following generalized eigenvalue problem

$$
[\widetilde{K}]\{\alpha\}=\lambda[\widetilde{M}]\{\alpha\} .
$$

The expressions of matrices $[\widetilde{M}]$ and $[\widetilde{K}]$ are given in the form of

$$
\begin{aligned}
& \widetilde{M}_{i j}=\varrho l \iint_{s_{h}} B_{i}(x, y) B_{j}(x, y) d s, \\
& \widetilde{K}_{i j}=D \iint_{s_{h}}\left\{\frac{\partial^{2} B_{i}}{\partial x^{2}} \frac{\partial^{2} B_{j}}{\partial x^{2}}+\frac{\partial^{2} B_{i}}{\partial y^{2}} \frac{\partial^{2} B_{j}}{\partial y^{2}}\right. \\
&+\nu\left(\frac{\partial^{2} B_{i}}{\partial x^{2}} \frac{\partial^{2} B_{j}}{\partial y^{2}}+\frac{\partial^{2} B_{i}}{\partial y^{2}} \frac{\partial^{2} B_{j}}{\partial x^{2}}\right) \\
&\left.+2(1-\nu) \frac{\partial^{2} B_{i}}{\partial x \partial y} \frac{\partial^{2} B_{j}}{\partial x \partial y}\right\} d s .
\end{aligned}
$$

By solving this eigenvalue problem, we can obtain the shape functions of dry-eigenmodes of the structure. The $r$-th eigenmode can be expressed in term of the $r$-th eigenvector $\left\{\alpha_{r}\right\}$ like

$$
u_{r}(x, y)=\sum_{l} \alpha_{r}^{l} B_{l}(x, y) .
$$

Similar to the conventional finite element method, various kinds of edge condition for the deflection can be enforced in this eigenvalue problem. For example, condition of simple supported boundary is

$$
W=0,
$$

while the condition of fixed boundary is

$$
\left.\begin{array}{rl}
W & =0 \\
\frac{\partial W}{\partial N} & =0
\end{array}\right\} \text {. }
$$

The difference between the proposed method and conventional finite element method is that the predictions of stresses, strains as well as the internal moments by present method are continuous over the whole plate, because bi-cubic B-spline function $B_{l}(x, y)$ has continuous second derivatives, and the resultant deflection $W$ has the same continuity too.

\section{Hydrodynamic Pressure}

Pressure distribution method is widely applied in the studies of VLFS. We also use this method in present study for determining the pressure distributions. We have velocity potential

$$
\phi(\boldsymbol{r})=-\iint_{s_{h}} p\left(\boldsymbol{r}^{\prime}\right) G^{\prime}\left(\boldsymbol{r}, \boldsymbol{r}^{\prime}\right) d s,
$$

where $\boldsymbol{r}=(x, y, z)$ and $\boldsymbol{r}^{\prime}=(\xi, \eta, 0)$ represent the field point and source point respectively. Green's function is given by

$$
G^{\prime}\left(\boldsymbol{r}, \boldsymbol{r}^{\prime}\right)=\frac{1}{2 \pi} \lim _{\mu \rightarrow 0} \int_{0}^{\infty} \frac{\cosh k \cdot(z+h) J_{0}(k: R) k}{k \cdot \sinh k \cdot h-(K-i, \mu) \cosh k \cdot h} d k,
$$

where $R=\sqrt{(x-\xi)^{2}+(y-\eta)^{2}}, h$ is the water depth and $J_{0}$ the Bessel Function of zero-th order. When $r$ is also on the bottom of the plate, Green's function has the form of $G\left(\boldsymbol{r}, \boldsymbol{r}^{\prime}\right)=$ $G^{\prime}\left(\left|r-r^{\prime}\right|\right)$. The pressure distributions on the bottom of the plate must satisfy equation

$$
\left.\begin{array}{rl}
p_{r}(\boldsymbol{r})+K \iint_{s_{h}} p_{\boldsymbol{r}}\left(\boldsymbol{r}^{\prime}\right) G\left(\left|\boldsymbol{r}-\boldsymbol{r}^{\prime}\right|\right) d s & =-u_{r}^{\prime}(\boldsymbol{r}) \\
p_{D}(\boldsymbol{r})+K \iint_{s_{h}} p_{D}\left(\boldsymbol{r}^{\prime}\right) G\left(\left|\boldsymbol{r}-\boldsymbol{r}^{\prime}\right|\right) d s & =u_{I}(\boldsymbol{r})
\end{array}\right\} .
$$

Notice that the wavelength of practical interest for VLFS is of order 0.01 , the usage of high order discretization is indispensable. High order B-spline Galerkin panel method has been proposed for potential flow problem first by Maniar \& Newman ${ }^{2)}$, the advantageous of their method has been proved again by Kashiwaga's work ${ }^{1)}$. Their method is also applied in present study. We discretize the unknown radiation pressure of the $r$-th mode by the fourth order B-spline function

$$
p_{r}(x, y)=\sum_{i} \beta_{r}^{i} B_{i}(x, y) .
$$

To obtain the $i$-th unknown polygon $\beta_{r}^{i}$, we substitute this expression into equation (26), multiply the equation by $B_{i}(x, y)$ and make an integration over $S_{h}$. This procedure produces a linear system of simultaneous equations

$$
\sum_{j}\left[\mathcal{\tau}_{i j}+K \mathcal{G}_{i j}\right] \beta_{r}^{j}=-\mathcal{W}_{i}
$$

where

$$
\begin{aligned}
& \mathcal{I}_{i j}=\iint_{S_{h}} B_{i}(x, y) B_{j}(x, y) d x d y, \\
& \mathcal{G}_{i j}=\iint_{S_{h}} B_{i}(x, y)\left[\iint_{S_{h}} B_{j}(\xi, \eta) G\left(\left|r-r^{\prime}\right|\right) d \xi d \eta\right] d x d y, \\
& \mathcal{W}_{i}=\iint_{s_{h}} B_{i}(x, y) w_{r}(x, y) d x d y .
\end{aligned}
$$

We can obtain pressure distributions $p_{r}$ by solving equation (28). The scattering pressure $p_{D}$ can be determined in exactly the same way.

\section{Numerical Procedure}

In present method, singular integration in term $\mathcal{G}_{i j}$ is estimated by using the adaptive numerical quadrature proposed by $X \ddot{u}$ et $a^{{ }^{8}}{ }^{8}$. Estimation of term $\mathcal{G}_{i j}$ seems to be the most CUP consuming part. For a general case, $N^{2}$ times of integration should be done if we discretize the plate into $N$ panels. While if the plate is a horizontal one and be discretized into the panels with exactly the same shape, only $N$ times of integration are necessary because Green's function (25) is a function of the horizontal distance $R$ between $r$ and $\boldsymbol{r}^{\prime}$. This reduction has been called "relative similarity relations" in Kashiwaga's work $^{1)}$. In fact, around $90 \%$ of CUP time is used for solving 
the linear system of simultaneous equations (28) itself.

\section{Results and Discussions}

To validate the proposed method, we have made a comparison between the theoretical predictions and the experimental data. The principal particulars of the structure used in the comparison are listed in Table 1. The model test had been carried out at the Ship Research Institute of Japan, and the experimental data have been published by Yago et al ${ }^{5}$. In our calculation, we discretized the plate into $80 \times 16$ panels, that means 320 panels are used on one fourth of the plate for determining the pressure distributions.

Table 1. Principal Particulars of the Testing Model

\begin{tabular}{|c|c|}
\hline Length & $9.75(\mathrm{~m})$ \\
\hline Breadth & $1.95(\mathrm{~m})$ \\
\hline Draft & $1.66 \times 10^{-2}(\mathrm{~m})$ \\
\hline Stiffness $E I$ & $1.788 \times 10^{3}\left(\mathrm{kgf} \cdot \mathrm{m}^{2}\right)$ \\
\hline Water Depth & $1.90(\mathrm{~m})$ \\
\hline
\end{tabular}

As the first part of the validation, the eigenmodes of the plate have been estimated by the proposed method and NASTRAN with LANCZON method. The same meshes are used in both calculations. The first three eigenmodes obtain by both methods are all the rigid modes. Fig. 2 shows the first nine elastic modes. Contour lines of the modes obtained by BSEM

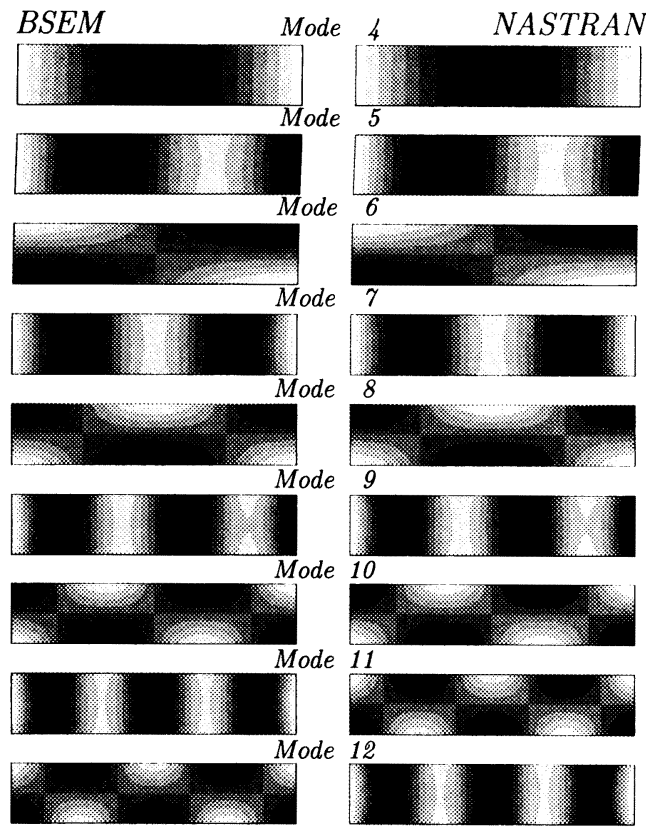

Fig. 2. Comparison of the elastic eigenmodes obtained by the proposed method to that by NASTRAN

are drawn on the left side of the figure, while those by NASTRAN are on the right side. There is no visible difference can be observed from this comparison, although modes 11 and 12 are in a reverse order by the different methods. Table 2 lists the natural frequencies of the first 20 elastic modes. Results in column BSEM are obtained by present method while those on column NAST are obtained by NASTRAN.
Table 2. Natural frequencies of some eigenmodes $\left(h_{z}\right)$

\begin{tabular}{|c|r|r||c|c|c|}
\hline Mode & BSEM & NAST & Mode & BSEM & NAST \\
\hline \hline 4 & 0.874 & 0.869 & 14 & 16.588 & 16.467 \\
\hline 5 & 2.420 & 2.406 & 15 & 20.246 & 19.992 \\
\hline 6 & 2.653 & 2.627 & 16 & 21.828 & 21.596 \\
\hline 7 & 4.768 & 4.739 & 17 & 23.037 & 22.710 \\
\hline 8 & 5.441 & 5.385 & 18 & 23.323 & 22.930 \\
\hline 9 & 7.915 & 7.864 & 19 & 25.326 & 24.989 \\
\hline 10 & 8.488 & 8.398 & 20 & 25.434 & 24.989 \\
\hline 11 & 11.860 & 11.775 & 21 & 27.948 & 27.495 \\
\hline 12 & 11.907 & 11.780 & 22 & 29.174 & 28.853 \\
\hline 13 & 15.797 & 15.611 & 23 & 31.090 & 30.654 \\
\hline
\end{tabular}

We can find that the difference of the natural frequency is very small, it increases from $0.57 \%$ of the first elastic modes to $1.4 \%$ of the 20 -th elastic mode. We can also find that the natural frequencies of mode 11 and 12 are so close that shows a reason of the order reverse. Generally speaking, the resultant eigenmodes depend on not only the shape of the plate but also the numerical discretization. Isoparametric elements are used in NASTRAN, while B-spline elements are used in the proposed method. Therefore differences between the eigenmodes obtained by the two methods should exist. These differences are found from higher eigenmodes, for example the 64-th eigenmode as shown in Fig. 3. Anyway it seems that the eigenmodes obtained by BSEM are reliable.
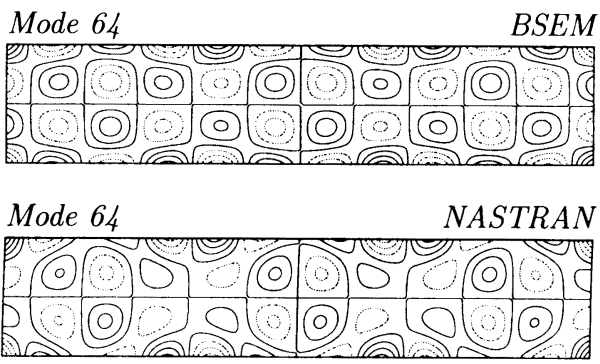

Fig. 3. Contour lines of the 64-th eigenmode

To compare with the experimental data, calculations have been done for the incident wave of $\lambda_{\infty} / L=0.1$ and $0.6\left(\lambda_{\infty}\right.$ is given by $g^{\prime} T^{2} / 2 \pi$, where $T$ is the period of the wavemaker). The directional angles are of $\chi=180^{\circ}, 210^{\circ}, 240^{\circ}$, and $270^{\circ}$. Fig. 4 shows the amplitudes of the first 120 eigenmodes in wave of wavelength ratio $\lambda_{\infty} / L=0.1$. We find that amplitude of
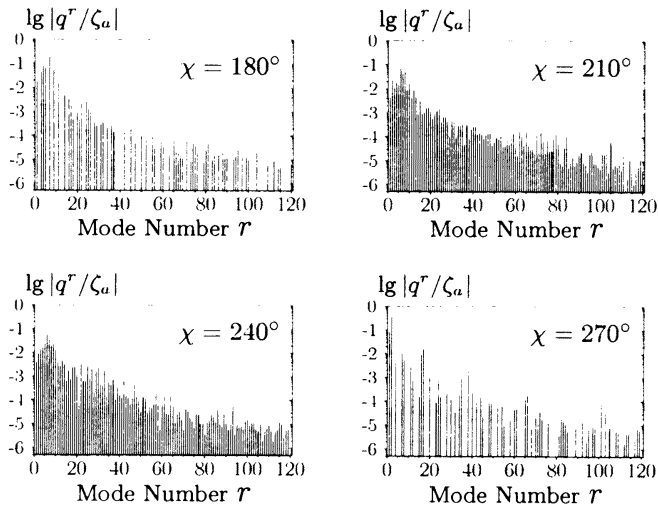

Fig. 4. Amplitudes of each eigenmodes in wave $\lambda_{\infty} / L=0.1$ 
the eigenmodes decreases exponentially with the mode number. We can also find that the modes antisymmetric about $x$-axis are not excited in head sea condition, while those antisymmetric about $y$-axis are not excited in beam sea condition. Considering the estimation of bending moment, we use 50 eigenmodes in the calculations of following comparisons.
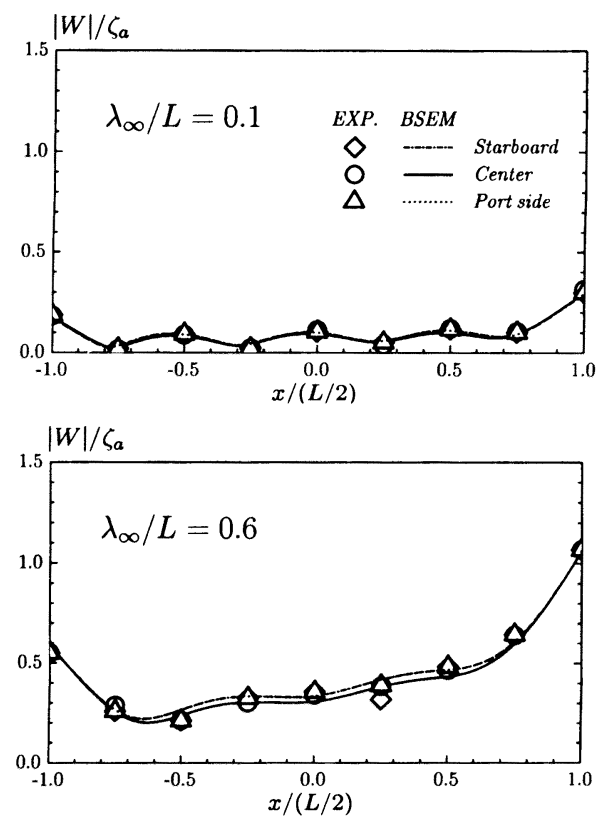

Fig. 5. Comparisons of the amplitude of vertical deflection in head sea condition $\left(\chi=180^{\circ}\right)$
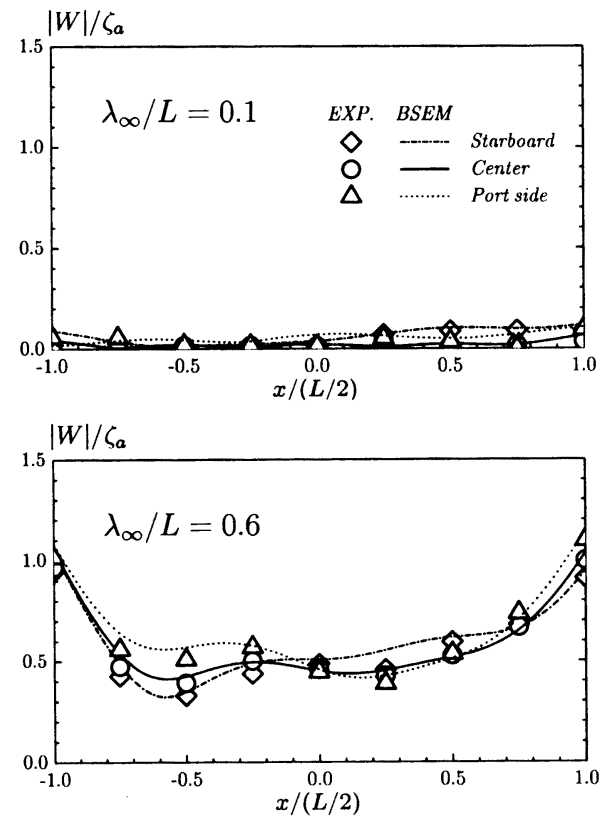

Fig. 6. Comparisons of the amplitude of vertical deflection in oblique sea condition $\left(\chi=210^{\circ}\right)$
First, we made a comparison for the amplitudes of deflections of the plate. We can see, from Fig. 5 to Fig. 8, that predictions by the proposed method agree with the experimental results very well in all of cases. We can also see that difference of the amplitudes between the center line and the rims of the plate exists even in the head sea and beam sea conditions.
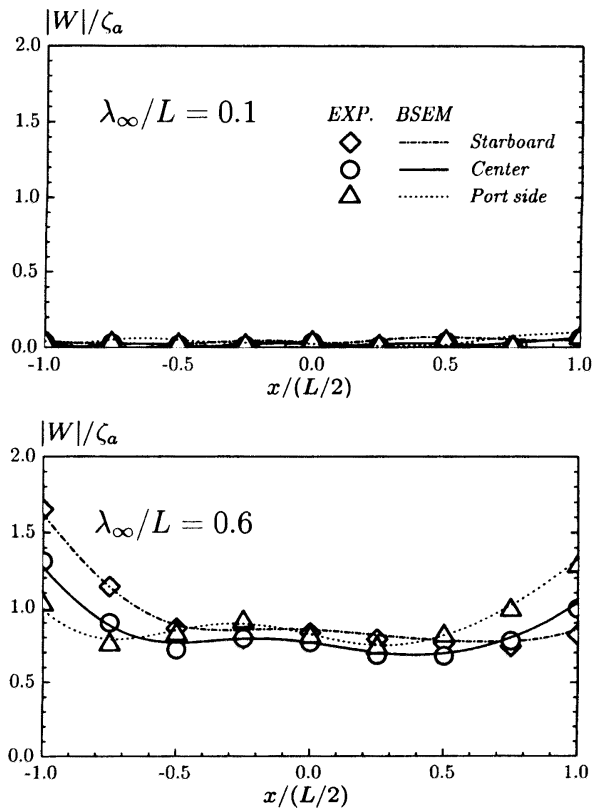

Fig. 7. Comparisons of the amplitude of vertical deflection in oblique sea condition $\left(\chi=240^{\circ}\right)$
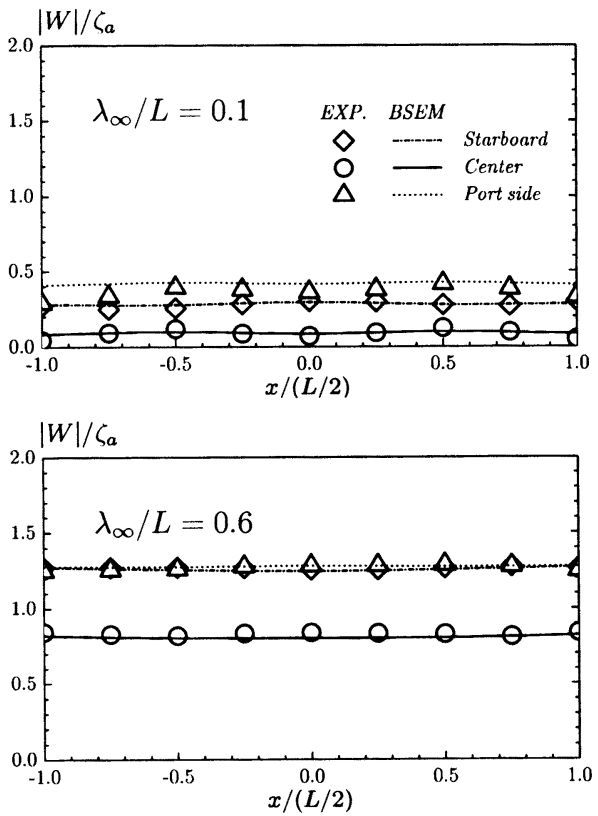

Fig. 8. Comparisons of the amplitude of vertical deflection in beam sea condition $\left(\chi=270^{\circ}\right)$ 
Next, we made a comparison of the bending moment $M_{x}$ in head sea condition $\left(\chi=180^{\circ}\right)$. Fig. 9 shows the amplitude of the bending moment along the center line. The results of theoretical calculations by the proposed method are in a very good agreement with the experimental data. From this figure we can find that the resultant bending moments decrease to zero at rims of the plate. It means that the boundary condition of the free-free plate has been satisfied by present method. Comparing the results of bending moment and deflection, we find that although maximum amplitude of the deflection of $\lambda_{\infty} / L=0.1$ is about $1 / 3$ of that of $\lambda_{\infty} / L=0.6$, but difference between the maximum bending moments of two cases is not so large. It reminds us that the bending moment may not be small in short wave cases though the deflection may.
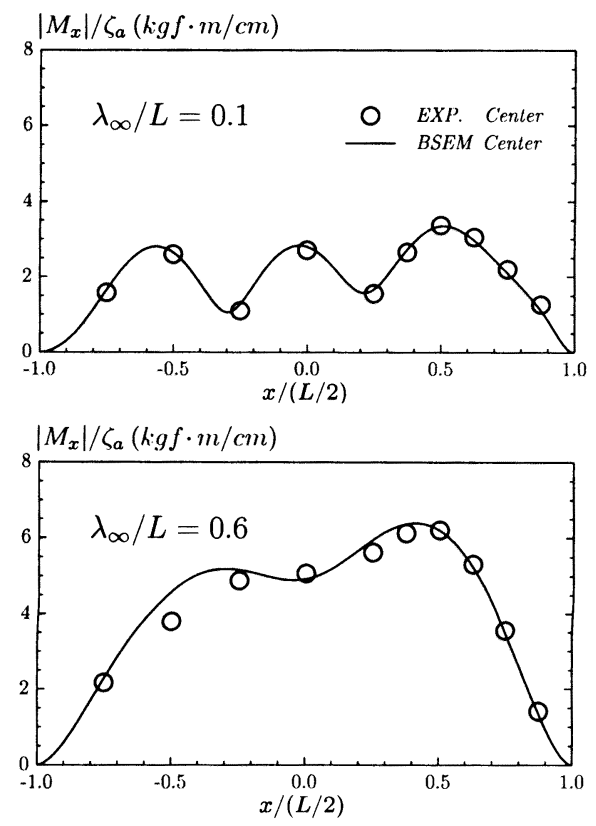

Fig. 9. Comparisons of the amplitude of bending moment $M_{x}$ in head sea condition $\left(\chi=180^{\circ}\right)$

The comparisons showed in Fig. 5 to Fig. 9 confirm the validity of the proposed method.

Finally, we apply the method to a realistic floating airport. The principal particulars of the structure are listed in Table 3. Incident wave condition is assumed of wavelength ratio $\lambda / L=$ 0.03 and wave directional angle $\chi=180^{\circ}$.

Table 3. Principal Particulars of the Calculated VLFS

\begin{tabular}{|c|cl|}
\hline \hline Length & 5000 & $(\mathrm{~m})$ \\
\hline Breadth & 1000 & $(\mathrm{~m})$ \\
\hline Draft & 5 & $(\mathrm{~m})$ \\
\hline Flexural Rigidity & $2.0 \times 10^{10}$ & $(\mathrm{k} g \mathrm{f} \cdot \mathrm{m})$ \\
\hline Water Depth & 50 & $(\mathrm{~m})$ \\
\hline
\end{tabular}

The resultant vertical deflection and pressure distributions are shown in Fig. 10. The real parts of the deflection and pressures are drawn on the left, the imaginary parts are on the center, and the amplitudes are on the right side of the figure. The non-dimensionalized deflection $w$ and total pressure $p$ are shown on the upper part while the non-dimensionalized diffraction pressure $p_{D}$ and radiation pressure $p_{R}$ are shown on the bottom part of the figure. Radiation pressure $p_{R}$ is defined by

$$
p_{R}=\sum_{r} \frac{q^{r}}{\zeta_{a}} p_{r}(x, y) .
$$

From the result of $p_{D}$, we can see that significant wave exciting pressures are on the tip of weather side of the plate although some small effects exist on the lee side and along the rims. We can also find that, in present case, incident wave with 33 wavelength on the plate excite a wavy elastic deflection of 14 wavelength, similar results has been found in Ohkusu's study ${ }^{3)}$. The deflection has a similar shape to the total pressure distribution, while the total pressure distribution seems to be dominated by the radiation pressure. Therefore we may say that it is very important to estimate the radiation pressure with reliable accuracy for predicting the hydroelastic motions of a VLFS.

\section{Concluding Remarks}

A B-spline element method has been proposed for predicting the hydroelastic responses of a very large floating structure in this study. Bi-cubic B-spline functions are used in the discretizations of both structure deflection and pressure distribution. The B-spline expressions of dry-eigenmodes of the structure are estimated by solving the eigenvalue problem of a motion equation that is proposed in this study according to the energy-conservation principle. Comparisons between the theoretical calculations and experimental data have been carried out for the vertical deflections and bending moments. The comparisons have shown a very good agreement between the results of calculations and experiments, and confirmed the reliability and accuracy of the proposed method.

\section{References}

1) Kashiwagi, M. and Furukawa,C., "A Mode Expansion Method for Predicting Hydroelastic Behavior of a ShallowDraft VLFS", Proc. of 16th Int. Conf. on Offshore Mesh. and Arc. Eng., Yokohama, Japan, pp. 179-186, (1997).

2) Maniar, H.D. and Newman, J.N., "Wave Diffraction by a Long Array of Circular Cylinders", 11th Workshop on Water Wave and Floating Bodies, Hamburg, Germany, (1996).

3) Ohkusu, M. and Nanba, Y., "Analysis of Hydroelastic Behavior of a Large Floating Platform of Thin Plate Configuration in Waves", Proc. of Int. Workshop on Very Large Floating Structures, Hayama, Japan, pp. 143-148, (1996).

4) Takaki, M. and Gu, X., "Motions of a Floating Elastic Plate in Waves", JSNAJ, Vol. 180, pp. 331-339, (1996).

5) Yago, K. and Endo, H., 1996, "On the Hydroelastic Response of Box-Shaped Floating Structure with Shallow Draft", JSNAJ, Vol. 180, pp. 341-352, (1996).

6) Maeda,H., Masuda,K., Miyajima, S. and Ikoma, I., "Hydroelastic Responses of Pontoon Type Very Large Floating Structure", JSNAJ, Vol. 178, pp. 203-212, (1995).

7) Mamidipudi, P. and Webster, W.C., "The Motion Performance of a Mat-like Floating Airport", Proc. of Int. Conf. on Hydroelasticity in Marine Technology, Norway, pp. 363-375, (1994).

8) Xü, H. and Yue,D.K.P., "Computations of Fully Nonlinear Three-Dimentional Water Waves", Proc. of 19th Symp. Naval Hydro., Seoul, Korea, (1992). 

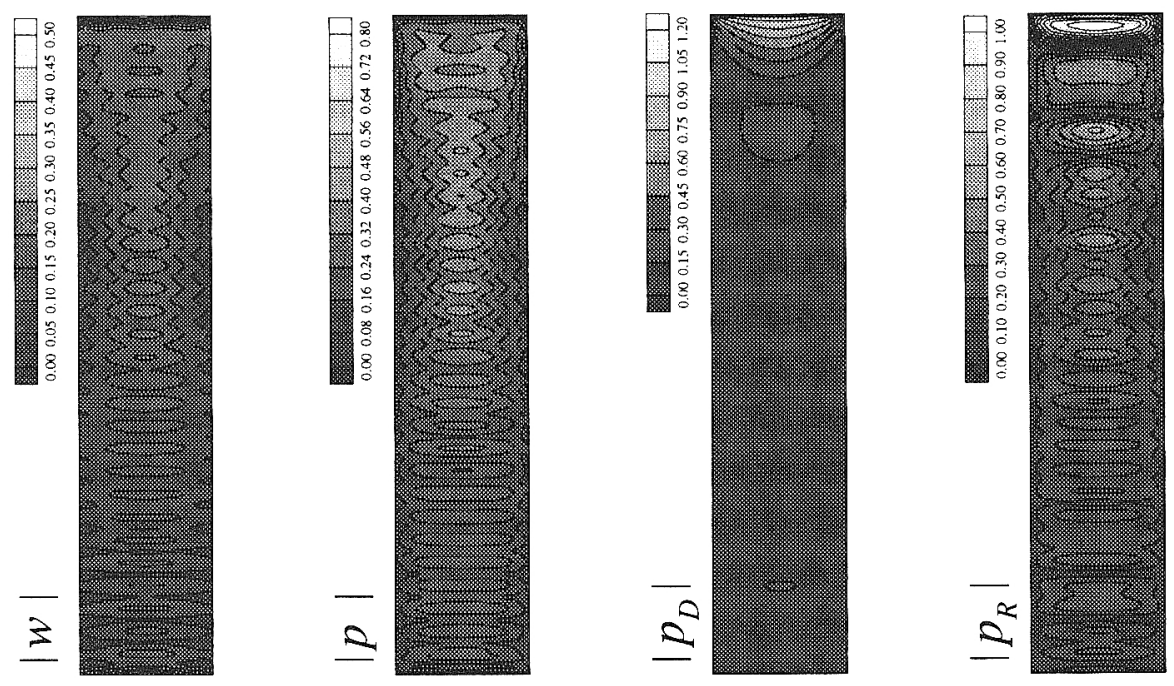

$\stackrel{\mathscr{E}}{\underline{E}}$
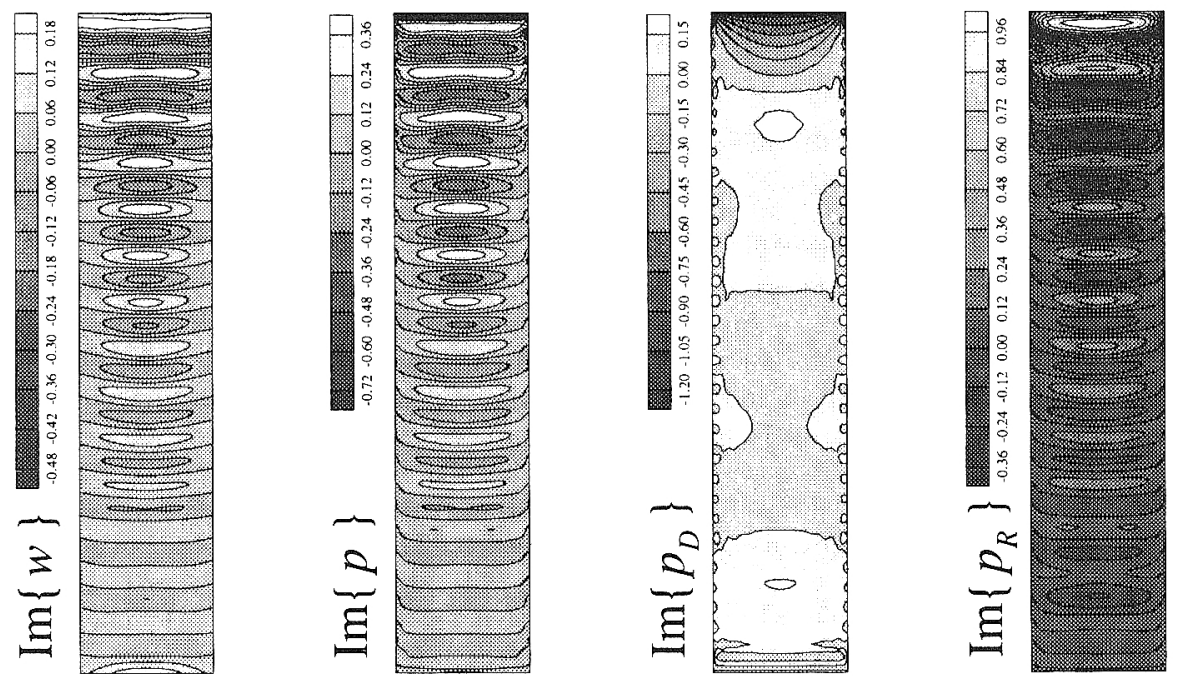

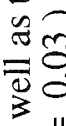

3

है

동

造

ॠ

है

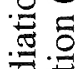

은

돈

世

응

象常

로

$\frac{5}{\sigma} \frac{0}{0}$

$\stackrel{2}{2}$

壱芯
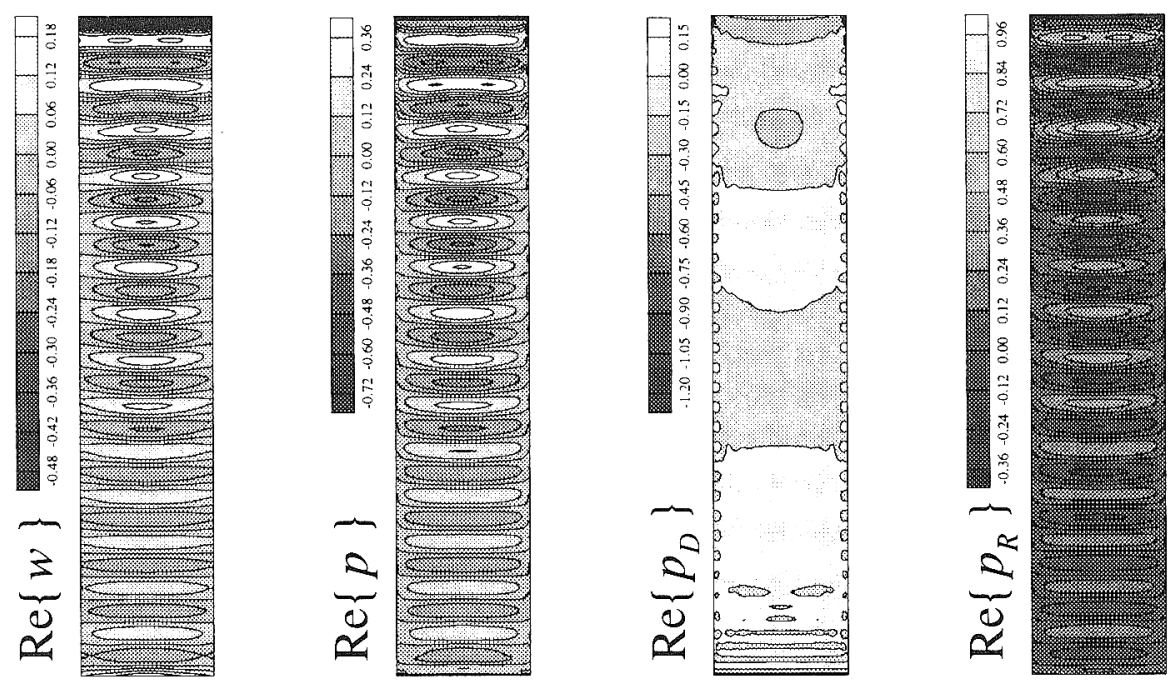

芯

.

음

造

ํㅗㅇ

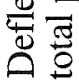

으

is 